\title{
Design and Implementation of Remote Automatic Detection of Tobacco Stack Data in Alcoholization Warehouse
}

\author{
Yanping Wang ${ }^{1, *}$, Hanxi $\mathrm{Li}^{1}$, Huan Le ${ }^{1}$, Lei Song ${ }^{1}$ and Shihai Tao ${ }^{2}$ \\ ${ }^{1}$ Hangzhou Cigarette Factory, China Tobacco, Zhejiang Industry Co. LTD, \\ No118, Kehai Road, Hangzhou, Zhejiang Prov. CHINA \\ ${ }^{2}$ Room 625, Haitai Building, No229, North $4^{\text {th }}$ Ring Road, Haidian, Beijing, CHINA \\ ${ }^{*}$ Corresponding author
}

\begin{abstract}
By remote extracting cycle sampling, the parameters of phosphine concentration, oxygen content and other important parameters will be tested in the tobacco stack, which not only ensures that the pressure in the pipe is reasonable, but also takes the pipe adsorption into full consideration, so as to ensure that the extracted gas can truly and effectively reflect the environmental parameters in the tobacco stack. The obtained parameters data are fully prepared for the establishment of big data analysis of tobacco storage and the improvement of online tobacco leaf alcoholization quality control and pests controlling.
\end{abstract}

Keywords-phosphine concentration; oxygen content; pipe adsorption; teflon; (key words)

The fumigation and disinfectant treatment with toxic gas such as phosphine is effective way to kill the tobacco pests in the tobacco warehouse, which is widely used in the storage period of tobacco alcoholization. The temperature and humidity and oxygen content indexes are very important in the process of tobacco leaf alcoholization.

\section{THE PROBLEM}

\section{A. Sampling Period}

In the mode of integration alcoholization, each tobacco stack has a small environment with different temperature, humidity, oxygen content based on storage area. Each PLC system is responsible for the rolls of each layer of 26 stack for data collection and analysis. Considering the time, accuracy, we will control the sampling period two hours or so, so each stack sampling time control within 5 minutes, including extraction time, balance time and sampling time.

\section{B. Sampling Pressure}

The pressure requirements of the sensors for the detection of phosphine and oxygen content are extremely demanding, and the pressure of one atmosphere is required to be $\pm 10 \%$ (that is, the pressure range of absolute pressure is $90 \sim 110 \mathrm{Kpa}$ ). Therefore, in the entire sampling process, it is necessary to ensure that the pressure is within the range of the sensor and enough scope for pressure fluctuation. It makes the extremely harsh choice of piping and the matching requirements with the sampling pump.

\section{Sampling Accuracy}

As it is a method to detect the parameters remotely by extracting the gas in the stack, the accuracy of the data collected is important for tobacco leaf alcoholization and pests controlling, and phosphine is a gas that is easy to absorb. So in the process of extraction, the influence on the gas parameters should be reduced as much as possible, the pipeline material selection is also important.

\section{SOLUTION}

A. System Principle

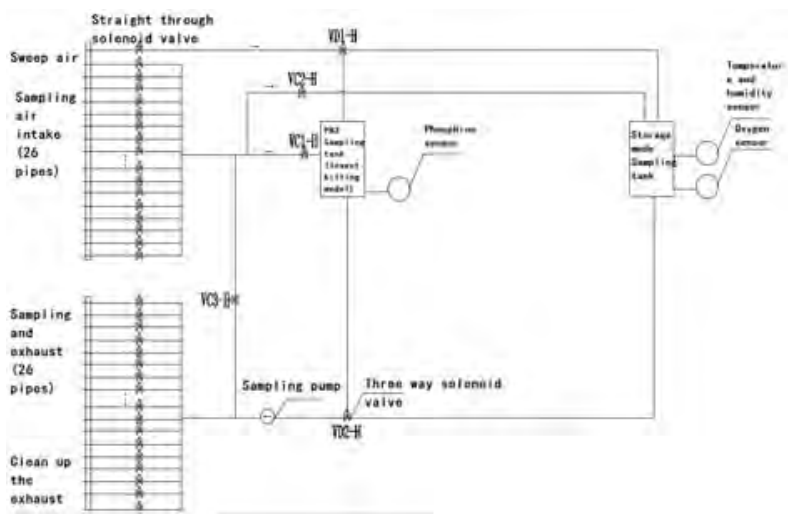

FIGURE I. PRINCIPLE

In this system, the parameters in the stack of 26 positions were detected by the sampling pump and the sampling tank, and the phosphine concentration in the killing pests mode and the oxygen content in the alcoholization mode were respectively detected by the switch of VD1-H and VD2-H three-way valves. Sample test is performed for each stack position by polling open and close valves.

\section{B. Sampling Period Calculation}

1). Pipe and tank volume calculation

Sampling tube diameter $8 \mathrm{~mm}$, inner diameter $6 \mathrm{~mm}$, The cross-sectional area of each tube is $\mathrm{S}=3.14 * 0.032=$ $0.002826 \mathrm{dm}^{2}$. According to the room layout, the longest 
pipeline is calculated as 100 meters. So, when all air is circulated through the pipe, the MAX air volume is $\mathrm{V}=\mathrm{S} * \mathrm{~L}=0.002826 * 1000=2.826 \mathrm{dm}^{3}$. The tank of detection is designed with $1.24 \mathrm{dm} * 1.14 \mathrm{dm} * 0.74 \mathrm{dm}$, and the volume is about $1.046 \mathrm{dm}^{3}$.

\section{2). Pipeline selection}

The principle of pipe diameter selection is to minimize the influence of sampling on tobacco stack, that is, to select the pipe with smaller diameter under the condition of reliable sampling. And materials are selected to minimize adsorption and ensure the accuracy of sampling concentration. After comprehensive consideration, the pipe with diameter of $8 \mathrm{~mm}$ and inner diameter of $6 \mathrm{~mm}$ is adopted, and the material is Teflon.

\section{3). Sampling time calculation}

The flow rate of the pump was $6 \mathrm{dm}^{3} / \mathrm{min}$, consideration of pipeline transmission loss, we calculate it with $4 \mathrm{dm}^{3} / \mathrm{min}$. The time for longest pipeline to the detection tank is $(2.826+1.046) / 4=0.968 \mathrm{~min}$, that means the system can extraction the gas less than $1 \mathrm{~min}$. In order to ensure the accuracy of sampling concentration, the system design takes 2 minutes.

\section{4). Sampling pressure calculation}

a) Internal resistance coefficient of pipe

The pipeline resistance coefficient of Teflon ( $\phi$ 8) is about $0.13-0.16$, according to the manual.

b) Pressure drop calculation $(\triangle \mathrm{P})$

$\Delta \mathrm{P}=\Delta \mathrm{P}_{f}+\Delta \mathrm{P}_{s}+\Delta \mathrm{P}_{N}$

$\triangle \mathrm{P}_{f}$ : frictional force pressure drop

$\triangle \mathrm{P}_{\mathrm{s}}$ : static pressure drop

$\triangle \mathrm{P}_{N}$ : speed pressure drop

$$
\Delta P_{f}=\left(\frac{\lambda L}{D}+\sum K\right) \frac{u^{2} \rho}{2} \times 10^{-3}
$$

The values in this project are as follows:

Coefficient of friction $\lambda=0.04$

Pipe inner dimeter $\mathrm{D}=0.006 \mathrm{dm}$

Average fluid velocity $\mu=3$

Fluid density $\rho=1.3$ (Air with PH3)

$\sum \mathrm{K}=0.2$

\section{Calculation:}

$\Delta \mathrm{P}_{f}=(0.04 * 100 / 0.006+0.2) * 9 * 1.3 * 0.001 / 2=3.901 \mathrm{kpa}$

That means when $\mathrm{L} \leq 100 \mathrm{~m}$, the pipeline pressure drop not much.

$\triangle$ Ps: pressure drop caused by height change of pipeline

$\triangle \mathrm{Ps}=2 * 1.2 * 9.81 * 10^{-3}=0.024 \mathrm{kpa}$ $\triangle \mathrm{P}_{\mathrm{N}}$ : pressure drop due to different inlet and outlet sections of a pipe or system resulting in changes in fluid flow rate.

$$
\Delta P_{N}=\frac{u_{2}^{2}-u_{1}^{2}}{2} \rho \times 10^{-3}
$$

$\mu_{1:}$ inlet fluid speed $\quad \mu_{2:}$ outlet fluid speed $(\mathrm{m} / \mathrm{s})$

$\rho:$ Fluid density $\left(\mathrm{kg} / \mathrm{m}^{3}\right)$

$\mu_{1}=3$ ( $\phi 6$ pipe) $\quad \mu_{2}=27$ ( $\phi 8$ pipe)

And then:

$$
\begin{aligned}
& \Delta \mathrm{P}_{N}=\left(27^{2}-3^{2}\right) * 1.2 * 10^{-3}=0.432 \mathrm{kpa} \\
& \begin{aligned}
\Delta \mathrm{P} & =\Delta \mathrm{P}_{f}+\Delta \mathrm{P}_{s}+\Delta \mathrm{P}_{N}=3.901+0.024+0.432=4.357 \mathrm{kpa} \\
& =0.04357 \mathrm{mbar}
\end{aligned}
\end{aligned}
$$

According to the pump flow and pressure drop curve, this pressure drop has little effect on the flow rate.

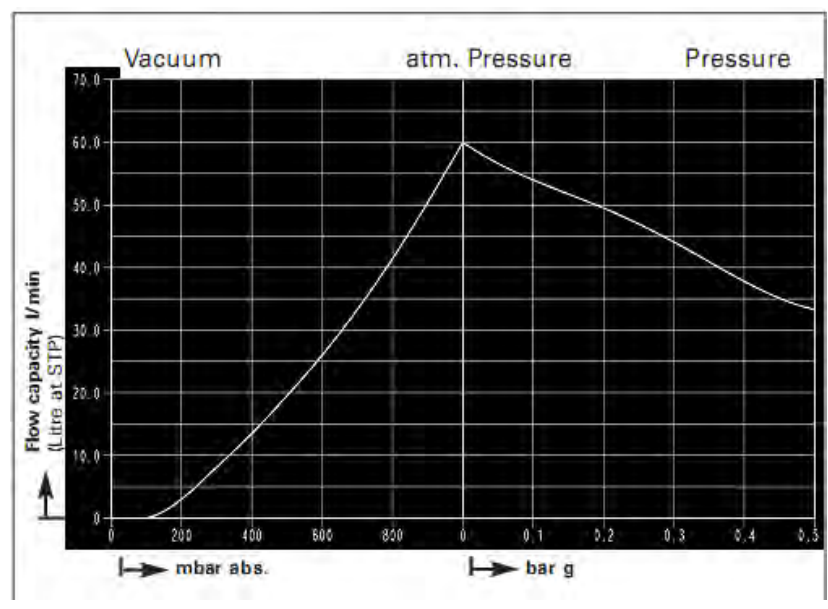

FIGURE II. FLOW AND PRESSURE DROP

\section{Control System}

The control logic is: [killing pest mode, to detect the $\mathrm{ph}_{3}$ ]

Start $\rightarrow$ system initializing $\rightarrow$ mode select $\rightarrow$ cleaning pipe and detection tank (1min) $\rightarrow$ sampling (auto mode) $\rightarrow$ first stack sampling (2min) $\rightarrow$ stop pump, balance and recording (1 min) $\rightarrow$ exhaust (2min) $\rightarrow$ th stack sampling $\rightarrow$ cycle to the 26th sampling.

When the recorded data exceeds the alert level, the system alerts. If system completes all the detection, it will cleaning all pipeline and detection tank to ensure the accuracy of next detection.

The principle of oxygen content detection is just like $\mathrm{ph}_{3}$ detection mode. Normally, there are 1-2 times killing pests by ph3 fumigation every year, every time continue about 10 days. In addition to the time of killing pests, other processes are alcoholization, the system change the mode to detect oxygen content in tobacco stack. 


\section{TEST RESULTS}

The system is set up in No.6 tobacco warehouse of Hangzhou Cigarette Factory. Select layout 3, B area, No.9 stack with random, system begin to detect the oxygen content. First we prepare oxygen fill in the stack, and we get the oxygen content value is $26.2 \%$ with handheld oxygen detection devices.

TABLE I. FIRST OXYGEN CONTENT RESULT

\begin{tabular}{|c|c|c|c|c|c|c|c|c|}
\hline Times (s) & 0 & 4 & 8 & 13 & 17 & 21 & 24 & 28 \\
\hline Concentration(\%) & 20.3 & 20.4 & 20.6 & 22.6 & 23.6 & 24.3 & 24.6 & 24.9 \\
\hline Times (s) & 32 & 36 & 39 & 47 & 52 & 59 & 65 & 70 \\
\hline Concentration(\%) & 25.1 & 25.6 & 25.7 & 25.9 & 26 & 26.1 & 26.2 & 26.2 \\
\hline
\end{tabular}

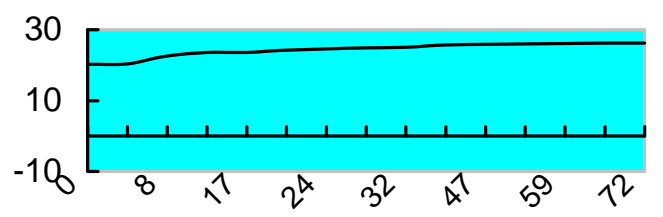

FIGURE III. TIME WITH CONCENTRATION

TABLE II. 2TH OXYGEN CONTENT RESULT

\begin{tabular}{|c|c|c|c|c|c|c|}
\hline Times (s) & 0 & 4 & 7 & 10 & 14 & 17 \\
\hline Concentration (\%) & 20.6 & 16.8 & 16.6 & 17.9 & 18.7 & 19.1 \\
\hline Times (s) & 20 & 24 & 27 & 30 & 33 & 39 \\
\hline Concentration (\%) & 19.7 & 20.0 & 20.3 & 20.4 & 21.1 & 22.0 \\
\hline Times (s) & 43 & 46 & 49 & 52 & 56 & 60 \\
\hline Concentration (\%) & 23.3 & 23.6 & 24.3 & 24.5 & 24.9 & 25.3 \\
\hline Times (s) & 65 & 70 & 73 & 80 & 89 & 100 \\
\hline Concentration (\%) & 25.5 & 25.7 & 25.8 & 25.9 & 26.0 & 26.2 \\
\hline
\end{tabular}

Oxygen content extraction time corresponds to concentration

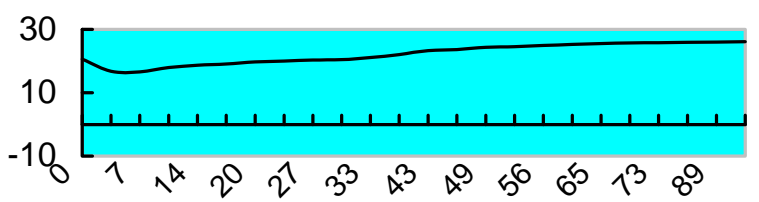

FIGURE IV. TIME WITH CONCENTRATION

TABLE III. 3TH OXYGEN CONTENT RESULT

\begin{tabular}{|c|c|c|c|c|c|c|}
\hline Times (s) & 0 & 10 & 15 & 18 & 22 & 26 \\
\hline Concentration (\%) & 20.5 & 17.1 & 17.8 & 18.6 & 19.4 & 19.9 \\
\hline Times (s) & 29 & 33 & 40 & 45 & 49 & 53 \\
\hline Concentration (\%) & 20.1 & 20.3 & 21.9 & 22.0 & $22 . .8$ & 23.1 \\
\hline Times (s) & 57 & 60 & 65 & 69 & 73 & 77 \\
\hline Concentration (\%) & 23.4 & 23.8 & 24.4 & 24.6 & 25.1 & 25.4 \\
\hline Times (s) & 82 & 86 & 92 & 96 & 100 & 104 \\
\hline Concentration (\%) & 25.5 & 25.6 & 25.8 & 25.9 & 26.0 & 26.1 \\
\hline
\end{tabular}

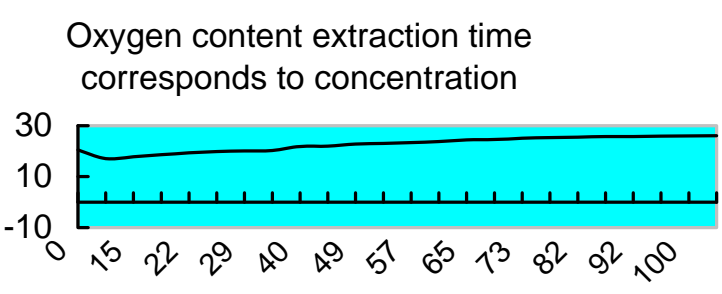

FIGURE V. TIME WITH CONCENTRATION

According to the data, we can carry out two-dimensional regression equation to obtain the relationship between concentration and time.

We get concentration (y) and time $(\mathrm{x})$ is:

$\mathrm{y}=16.039+0.10079 \mathrm{x}+0.00224 \mathrm{x}^{2}-$ $0.0000316 \mathrm{x}^{3}+0.00000008818 \mathrm{x}^{4}$

The formula can be used to approximately calculate the concentration in the tank within each time period, so that the time of the concentration balance in the tank and the corresponding concentration can be accurately grasped.

Other gas, such as phosphine, have the approximate results by experiment and calculation. It proves that the initial design of 2 minutes to achieve the same concentration as tobacco stack is credible.

\section{CONCLUSION}

The detection of phosphine in the fumigation and insecticidal process of tobacco leaves, and the detection of temperature and humidity in tobacco stack process and the detection of oxygen content are mostly performed manually by operators in China at present. On the one hand, it is dangerous to detect the concentration of phosphine at the scene; on the other hand, it is impossible to monitor the concentration of phosphine in real time, not to mention that the tobacco warehouse is large and requires a lot of detection.

This online monitoring system improves the automation and informatization of tobacco alcoholization information collection and tobacco leaf maintenance management, improves the timeliness and data accuracy, saves human resources, and realizes the monitoring and management. The system integrated with measurement, data collection, online monitoring and management. And we have a good foundation for the integrated management of digital tobacco alcoholization system.

\section{REFERENCE}

[1] Daxian CHENG, Mechanical design manual, the Fifth Edition [M] Chemical Industry Press. pp. 1-9, 2008.

[2] Zurong DING, Fluid mechanics [M], Higher Education Press, Dec. 2003, pp.20-102.

[3] Zengji CAI and Tianyu LONG, Fluid mechanics,pump and fan[M], China Construction Industry Press Dec. 1999, pp. 90-103.

[4] Sirong ZHANG and Yanping WANG Innovation and practice of modern cigarette factory construction [M], China Construction Industry Press. May 2014, pp. 299-314. 
[5] Yanping WANG and Hanxi LI The design and implementation of high precision and low power wireless temperature sensor, 2016 International Conference on Communications Information Management and Network Security sep.2016, pp59-64.

[6] Tobacco storage and maintenance [S]. Gas controlled storage. China tobacco industry standards, YC/T 322-2009.

[7] Tobacco storage and maintenance [S]. General technical requirements China tobacco industry standards, YC/T 322-2009.

[8] Occupational exposure limit for hazardous agents in the workspace Chemcial hazardous agents [S]. China national standards, GBZ2.1-2007. 